\title{
Symmetry energies and the curvature of the nuclear mass surface
}

\author{
J. Jänecke ${ }^{1, a}$ and T.W. O’Donnell ${ }^{2}$ \\ 1 Department of Physics, University of Michigan, Ann Arbor, MI 48109-1120, USA \\ 2 Michigan Center for Theoretical Physics and Science, Technology and Society Program, Residential College, University of \\ Michigan, Ann Arbor, MI 48109-1245, USA
}

Received: 24 November 2004 / Revised version: 16 February 2005 /

Published online: 20 April 2005 - (C) Società Italiana di Fisica / Springer-Verlag 2005

\begin{abstract}
A global study of the symmetry energies reflects upon the curvature of the mass surface. Special attention is given to the region from ${ }^{56} \mathrm{Ni}$ to ${ }^{100} \mathrm{Sn}$. Isospin inversion is indicated for odd-odd self-conjugate nuclei. Coexistence of isoscalar and isovector $n-p$ interactions in the localized region $N \approx Z, A=76-96$ is suggested by the isospin dependence of the symmetry energy. Experimental symmetry energies and values extracted from nine mass equations are compared. Overall agreement exists, but some distinct differences are also observed.
\end{abstract}

PACS. 21.10.Dr Binding energies and masses - 21.60.-n Nuclear structure models and methods

\section{Introduction}

Symmetry energies $E_{\text {sym }}(A, T)$ depend strongly on isospin $T$ and also on nucleon number $A$. They influence the curvatures of the experimental nuclear mass surface as well as the mass surfaces obtained from mass equations. The goodness of mass equations can therefore be tested by comparing the calculated and experimental values.

\section{Symmetry energies}

Excitation energy differences between isobaric analog states with isospins $T^{\prime}$ and $T$ in nuclei with nucleon number $A$ are denoted by $\Delta_{T^{\prime}, T}(A)$. While many such energies have been measured directly, a set including all known nuclei was used in the present work. It was deduced from Coulomb-energy-corrected differences of all available experimental masses for neighboring isobars.

The energies $\Delta_{T^{\prime}, T}(A)$ can furthermore be expressed as differences between symmetry and pairing energies $[1,2]$. An expression for the symmetry energies has been introduced as

$$
E_{\mathrm{sym}}(A, T)=\frac{a(A, T)}{A} T(T+1) .
$$

The factor $a(A, T)$ is an operationally defined symmetry energy coefficient. Symmetry energies $E_{\mathrm{sym}}(A, T)$ and the coefficients $a(A, T)$ were deduced globally over the entire bregion of experimentally known nuclei from

$$
a(A, T)=\frac{A \Delta_{T+2, T}(A)}{4 T+6} \quad \text { for } A=\text { even and odd }
$$

which is valid for both odd- $A$ and even- $A$ nuclei. The energies $\Delta_{T+2, T}(A)$ were obtained with the use of the new updated atomic mass evaluation Ame2003 [3].

\section{Results}

Results were discussed earlier [1,2]. The symmetry energy coefficients $a(A, T)$ are nearly constant over wide ranges of nuclei where the shell model dominates in the description of the symmetry energies. Systematic deviations from a constant value are observed, though, particularly for shell regions where neutrons and protons occupy different shellmodel orbits. Furthermore, an interesting effect was observed locally for nuclei with $N \approx Z$ in the region $A=76$ to $96[4]$.

The quantity $a(A, T)$ displays an essentially smooth dependence on $A$ for most of the $f p g$ shell containing the $p_{1 / 2} p_{3 / 2} f_{5 / 2} g_{9 / 2}$ shell-model orbitals. The nuclei with $N=Z$ display a particularly interesting behavior. Here, the even-even self-conjugate nuclei from $A=58$ to 98 follow a smooth dependence on mass number $A$. These nuclei have $T=0$ ground states. For the odd-odd nuclei with $N=Z$ with ground states of $T=0$ or $T=1$, however, anomalies are observed for $A=62,66,70$, and 74 suggesting isospin inversion in agreement with experiment. The departures from the smooth $T=0$ curve make it possible to estimate the excitation energies for the lowest $T=0$ states [4].

The symmetry energies of most nuclei in the $f p g$ shell with $Z>28$ and $N<50$ follow a $T(T+1)$ dependence on isospin as expected for the shell model. Distinct local deviations in the heavier region near $N=Z$ from

\footnotetext{
a e-mail: janecke@umich.edu
} 


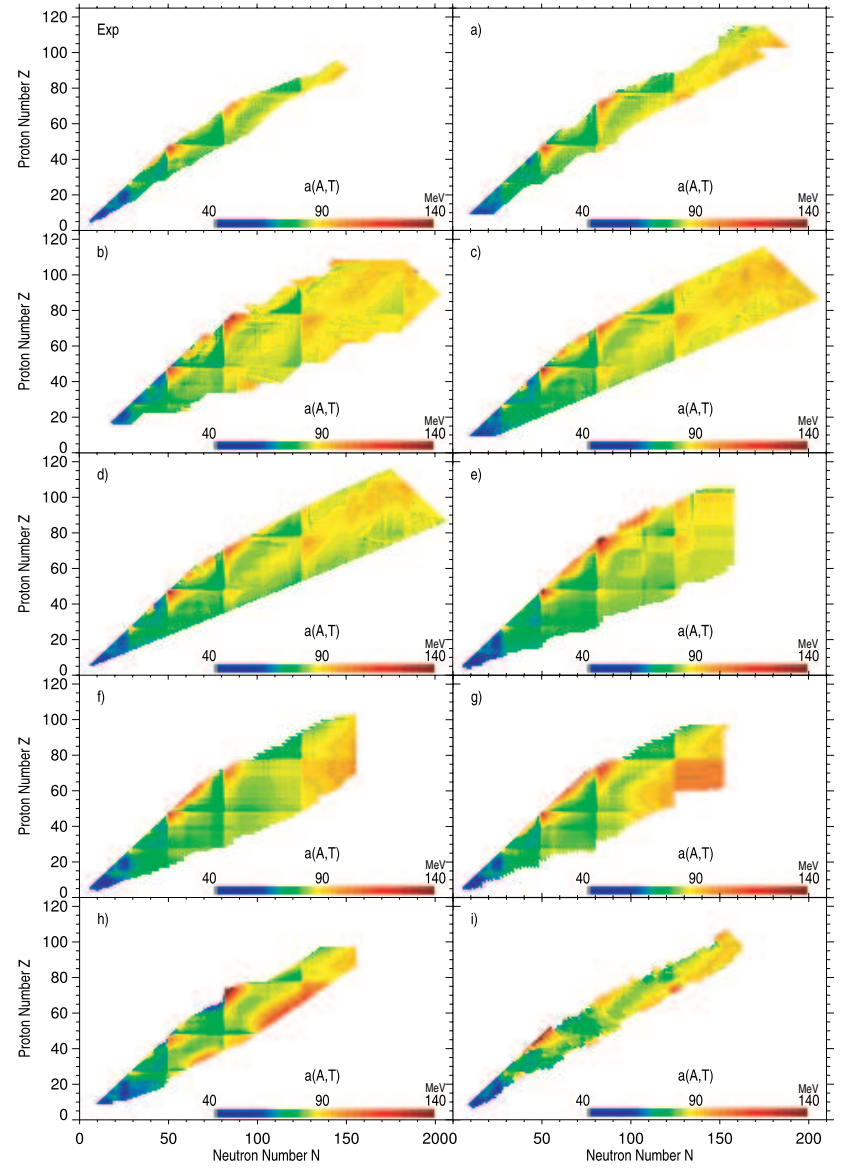

Fig. 1. Symmetry-energy coefficients $a(A, T)$ as a function of neutron and proton numbers $N$ and $Z$ deduced from experimental mass data and from mass equations or algorithms: (a) ref. [5], (b) ref. [6], (c) ref. [7], (d) ref. [8], (e) ref. [9], (f) ref. [10], (g) ref. [11], (h) ref. [12], (i) ref. [13].

$A=76$ to 96 and centered at $A \approx 86$ are observed. Here, the symmetry energies display a $T(T+4)$ dependence on isospin. Such a behavior is compatible with the Wigner supermultiplet model [14], and contributions from the isovector and isoscalar $n-p$ interactions are thus indicated. However, spin-isospin symmetry is known to be broken in heavier nuclei contrary to an interpretation in terms of $S U(4)$ spin-isospin symmetry. Lunney, Pearson, and Thibault [15] pointed out that the isoscalar $n-p$ interaction appears to provide a more direct description of this localized effect. The intense interest in the $T=0$ $n-p$ interaction is reflected in the numerous theoretical approaches reported in the literature (see, e.g., references cited in ref. [4]). The origin of the above effect is not entirely understood.

\section{Mass equations}

Replacing the experimental mass data by available theoretical mass predictions as basis for the above procedures to extract symmetry energies makes it possible to directly compare theoretical and experimental quantities, particularly the symmetry energy coefficients $a(A, T)$. Such a comparison reflects upon the goodness or possible shortcomings of the respective mass equation. A study of so far nine mass equations or procedures for reproducing experimental masses and extrapolating into regions of unknown nuclei is in progress [16].

While approximate agreement exists, as seen in fig. 1, distinct discrepancies between theoretical and experimental quantities are observed particularly in regions of neutron-rich and proton-rich nuclei. The unusual behavior of the mass surface characterized by the $T(T+4)$ dependence of the symmetry energy in the upper fpg shell near $N \approx Z$ is apparently not reproduced except, it seems, for mass equation (g). Interestingly, some mass equations appear to predict a similar behavior for nuclei approaching $N=Z$ for the next higher shell from $A=100$ to $A=164$. Mass equation (h) gives poor extrapolations for heavier very poton-rich and neutron-rich nuclei. Mass equation (i) displays no shell effects for both, the symmetry energy coefficients $a(A, T)$ and the pairing energies $P(A, T)$. Many additional details become apparent from the comparison of the curvature of the experimental and theoretical mass surfaces, both, in the region of nuclei which overlaps with the experimentally known masses and for the extrapolated regions. These effects become more apparent by displaying (not shown) the differences between the theoretical and experimental values.

\section{References}

1. J. Jänecke, T.W. O'Donnell, V.I. Goldanskii, Phys. Rev. C 66, 024327 (2002).

2. J. Jänecke, T.W. O'Donnell, V.I. Goldanskii, Nucl. Phys. A 728, 23 (2003).

3. G. Audi, A.H. Wapstra, C. Thibault, Nucl. Phys. A 729, 337 (2003).

4. J. Jänecke, T.W. O'Donnell, Phys. Lett. B 605, 87 (2005).

5. P. Möller, J.R. Nix, At. Data Nucl. Data Tables 39, 213 (1988).

6. Y. Aboussir, J.M. Pearson, A.K. Dutta, F. Tondeur, Nucl. Phys. A 549, 155 (1992).

7. P. Möller, J.R. Nix, W.D. Myers, W.J. Swiatecki, At. Data Nucl. Data Tables 59, 185 (1995).

8. W.D. Myers, W.J. Swiatecki, Nucl. Phys. A 601, 141 (1996).

9. T. Tachibana, M. Uno, M. Yamada, S. Yamada, At. Data Nucl. Data Tables 39, 251 (1988).

10. E. Comay, I. Kelson, A. Zidon, At. Data Nucl. Data Tables 39, 235 (1988).

11. J. Jänecke, P.J. Masson, At. Data Nucl. Data Tables 39, 265 (1988).

12. P.J. Masson, J. Jänecke, At. Data Nucl. Data Tables 39, 273 (1988).

13. L. Satpathy, R.C. Nayak, At. Data Nucl. Data Tables 39, 241 (1988).

14. E.P. Wigner, Phys. Rev. 51, 106 (1937).

15. D. Lunney, J.M. Pearson, C. Thibault, Rev. Mod. Phys. 75, 1021 (2003).

16. J. Jänecke, T.W. O'Donnell, in preparation. 